\title{
Primary Pulmonary Liposarcoma with Pancreatic Metastasis: A Rarest of Rare Intrathoracic Malignancy
}

\begin{abstract}
Liposarcoma of the lung is an extremely rare malignancy with $<20$ cases reported so far. With such limited evidence, natural history and appropriate management of this unusual tumor is unclear. We experienced a case of primary pulmonary myxoid liposarcoma in a 34-year-old male who presented with multiple distant site metastases including pancreas, which is again an extremely rare finding accounting for approximately $2 \%$ of pancreatic malignancies. Indexed case was treated with systemic chemotherapy.
\end{abstract}

Keywords: Lung primary, myxoid liposarcoma, pancreatic metastasis

\section{Introduction}

Primary pulmonary liposarcoma is an extremely rare malignancy with $<20$ cases reported in literature. ${ }^{[1,2]}$ Intrathoracic liposarcomas are uncommon, and if at all encountered, are usually located in the mediastinum. ${ }^{[3]}$ Malignant degeneration of a pulmonary lipoma and pleuropulmonary asbestosis have been considered as possible pathogenetic factors. ${ }^{[4]} \mathrm{We}$ herein present a widely metastatic case of primary pulmonary liposarcoma in a 34-year-old man who was treated with palliative chemotherapy.

\section{Case Report}

An otherwise well, 34-year-old male with no history of tobacco addiction presented with hemoptysis, intermittent fever, and significant weight loss for 1 year. Examination revealed a $5 \mathrm{~cm} \times 4 \mathrm{~cm}$ right axillary lymph node and decreased breath sounds over the left upper lung region, apart from which no other abnormality was detected. Contrast-enhanced computed tomography (CT) of the thorax showed a large neoplasm adjacent to bifurcation of the left main bronchus causing complete collapse of the left lung [Figure 1]. CT-guided biopsy from the lung mass was done for histopathological examination (HPE),

This is an open access journal, and articles are distributed under the terms of the Creative Commons Attribution-NonCommercial-ShareAlike 4.0 License, which allows others to remix, tweak, and build upon the work non-commercially, as long as appropriate credit is given and the new creations are licensed under the identical terms.

For reprints contact: WKHLRPMedknow_reprints@wolterskluwer.com which showed features of high-grade myxoid liposarcoma [Figure $2 \mathrm{a}$ and b]. Tumor cells were positive for $\mathrm{S} 100$ and $\mathrm{Ki}$ 67 was $15 \%$. ${ }^{18}$ F-fluorodeoxyglucose (FDG) positron emission tomography (PET)-CT scan revealed a mild hypermetabolic predominantly hypodense cystic lesion seen involving the left lung upper lobe inferiorly extending up to the left hilar region, causing abrupt cut-off of main bronchus and encasing upper and lower lobe bronchi causing distal complete collapse consolidation of the left lung upper and lower lobes [Figure 3a]. Mildly FDG-avid metastatic sites included bilateral axillary lymph nodes, right latissimus dorsi muscle, and hypodense cystic lesion involving pancreas [Figure $3 \mathrm{~b}$ and $\mathrm{c}$ ]. In lieu of widely metastatic disease, he was started on palliative chemotherapy with MAID regimen consisting of MESNA, adriamycin, ifosfamide, and dacarbazine chemotherapeutic agents.

\section{Discussion}

Liposarcoma is the most common mesenchymal tumor in adults. ${ }^{[5]}$ Most frequent occurrence is seen in the retroperitoneal space or in the lower extremities. The primary thoracic liposarcoma is unusual. If reported, it usually arises from mediastinum. ${ }^{[5]}$ Exhaustive literature search suggests that fewer than twenty cases have so far been reported. ${ }^{[1,2]}$ It shows

\footnotetext{
How to cite this article: Halder A, Biswas R, Shukla S, Rana N, Yadav V, Kaur J. Primary pulmonary liposarcoma with pancreatic metastasis: A rarest of rare intrathoracic malignancy. Indian $\mathrm{J}$ Med Paediatr Oncol 2020;41:605-7.
}

\section{Anirban Halder ${ }^{1}$, Rituparna Biswas', Sujit Shukla ${ }^{1}$, Nisha Rana ${ }^{3}$, Vikas Yadav ${ }^{1}$, Jaspreet Kaur ${ }^{1}$}

${ }^{1}$ Department of Radiation Oncology, VMMC and Safdarjung Hospital,

New Delhi, India, ${ }^{2}$ Department of Radiation Oncology, Dr. BRA-IRCH, All India Institute of Medical Sciences, New Delhi, India, ${ }^{3}$ Department of Pathology, VMMC and Safdarjung Hospital, New Delhi, India

Submitted: 12-May-2019 Revised: 29-Dec-2019 Accepted: 03-Jan-2020 Published: 29-Aug-2020

Address for correspondence: Dr. Rituparna Biswas, Department of Radiation Oncology, Dr. BRA-IRCH, All India Institute of Medical Sciences, New Delhi, India. E-mail: mail4r_biswas@ yahoo.co.in

Access this article online Website: www.ijmpo.org DOI: 10.4103/ijmpo.ijmpo_114_19 Quick Response Code:

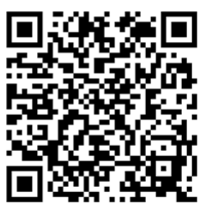




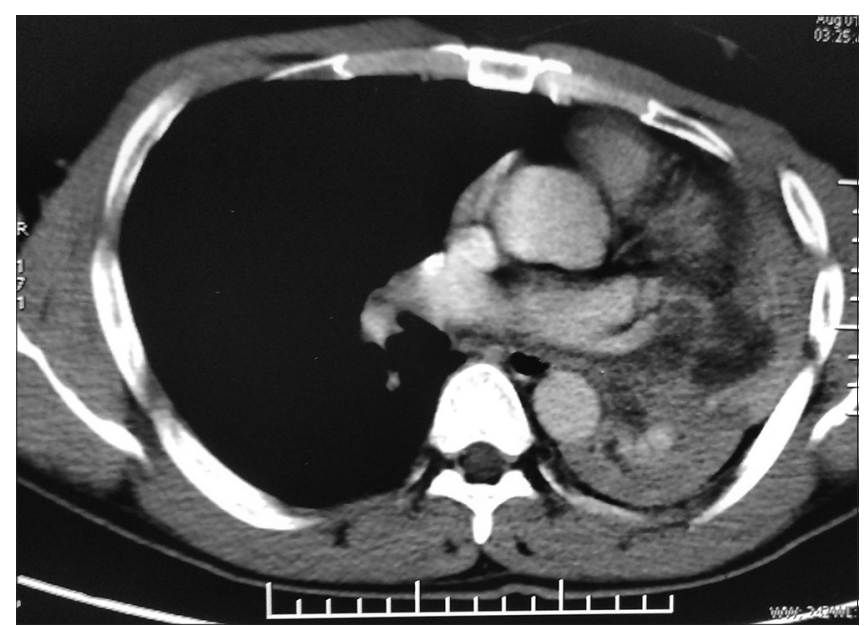

Figure 1: Contrast-enhanced computed tomography of the thorax showed a large neoplasm adjacent to bifurcation of the left main bronchus causing complete collapse of the left lung

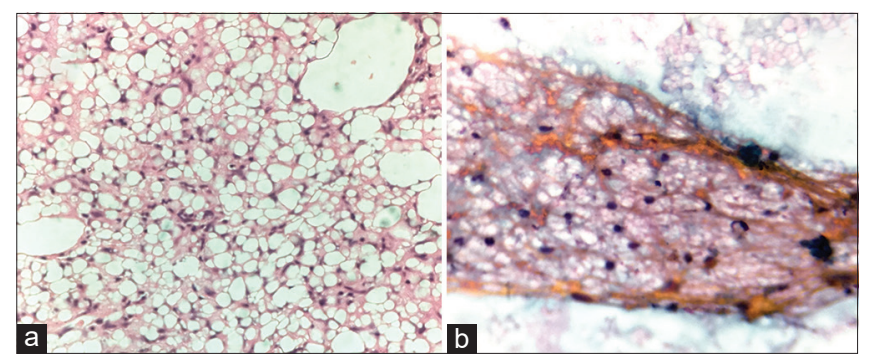

Figure 2: (a) High power $(\times 400)$ view showing sheets of variable-sized cells having sharply demarcated cytoplasmic vacuole and eccentrically placed hyperchromatic nuclei, lipoblasts (black arrow) present within the interalveolar space. (b) Cytology smear showing clusters of cells with vacuolated cytoplasm and hyperchromatic nuclei

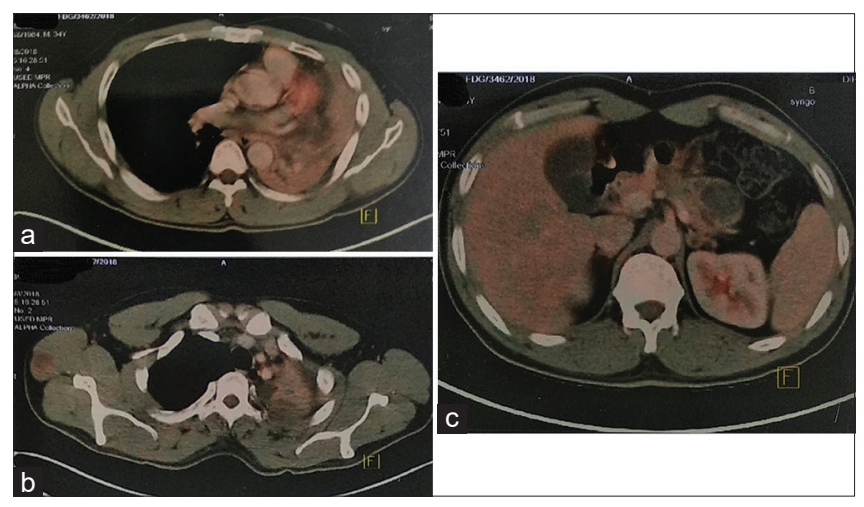

Figure 3: (a) Fluorodeoxyglucose positron emission tomography-computed tomography scan revealed a mild hypermetabolic predominantly hypodense cystic lesion seen involving the left lung upper lobe, (b) Mildly fluorodeoxyglucose avid metastatic deposit is seen in the right latissimus dorsi muscle and (c) Mildly fluorodeoxyglucose-avid hypodense cystic lesion involving pancreas

a slight male predilection as seen in our case too with reported patient age range of $9-59$ years. ${ }^{[5,6]}$ The presenting symptoms simulate that of lung tumor such as dry cough or with expectoration, hemoptysis, chest pain, fever, dyspnea, reduced appetite and general weakness, so on and so forth. There are no special characteristic symptoms and signs attributable to sarcoma of the lung, similarly as is seen in our case. Liposarcoma is subclassified histologically into well-differentiated, myxoid, round-cell, pleomorphic, dedifferentiated, and mixed types. ${ }^{[5]}$ After careful HPE of the index liposarcoma case, we considered that the cell differentiation, the mitotic index, presence of necrosis, and the proliferative activity of the tumor cells, defined the lesion as high-grade myxoid liposarcoma. Myxoid liposarcoma typically presents as a slow-growing, large mass and remains asymptomatic for several years; although there is a risk of recurrence, chance of metastases seems rare. ${ }^{[5,7]}$ On the contrary, our index case presented with upfront widespread metastases including pancreas which is anyhow an extremely rare finding accounting for approximately $2 \%$ of pancreatic malignancies, hence featuring our presented case immensely unique. ${ }^{[8]}$ However, metastases to the lung, pleura, liver, bone, kidneys, adrenal glands, and mediastinal lymph nodes have been reported for this category of tumor. ${ }^{[7]}$ This tumor presents difficult diagnostic dilemmas pertaining to pathology and CT appearances. ${ }^{[5]}$ Distinction of pulmonary liposarcoma from mediastinal variant requires careful radiographic evaluation, surgical evaluation, or both. Although definite conclusion regarding its appropriate management is difficult to draw from these limited evidences, based on information from all previously published reports, it appears that patients with primary pulmonary liposarcoma may benefit most from adequate surgical resection combined with radiation therapy. ${ }^{[6]}$ Adjuvant irradiation at a dose level of 60-66 Gy after wide local excision has been shown to improve the local control rate and probably the overall survival in lot of patients. ${ }^{[9]}$ Tumor recurrence is common in cases where complete surgical excision with clear margins was not possible. Chemotherapy has been used in both adjuvant and palliative settings, with highest activity observed with ifosfamide, anthracyclines, and dacarbazine based chemotherapy. ${ }^{[10]}$

\section{Conclusion}

The primary pulmonary liposarcoma is an extremely rare tumor with potential of local complications and distant metastases. The outcome depends on the precise pathologic diagnosis, stage at diagnosis, the adequacy of surgical intervention, and the use of adjuvant therapy in the form of radiotherapy and/or chemotherapy. Systemic chemotherapy has to be employed in metastatic settings.

\section{Declaration of patient consent}

The authors certify that they have obtained all appropriate patient consent forms. In the form the patient(s) has/have given his/her/their consent for his/ her/their images and other clinical information to be reported in the journal. The patients understand that their names and initials will not be published and due efforts will be made to conceal their identity, but anonymity cannot be guaranteed. 


\section{Financial support and sponsorship}

Nil.

\section{Conflicts of interest}

There are no conflicts of interest.

\section{References}

1. Longano A, DuGuesclin A, Mitchell C. Primary dedifferentiated liposarcoma of the lung with rhabdomyoblastic and chrondroblastic differentiation. Histopathology 2015;67:923-5.

2. Son C, Choi PJ, Roh MS. Primary pulmonary myxoid liposarcoma with translocation $t(12 ; 16)(q 13 ; p 11)$ in a young female patient: A brief case report. Korean J Pathol 2012;46:392-4.

3. Krygier G, Amado A, Salisbury S, Fernandez I, Maedo N, Vazquez T. Primary lung liposarcoma. Lung Cancer 1997;17:271-5.

4. Giménez A, Franquet T, Prats R, Estrada P, Villalba J, Bagué S.
Unusual primary lung tumors: A radiologic-pathologic overview. Radiographics 2002;22:601-19.

5. Uchikov A, Poriazova E, Zaprianov Z, Markova D. Low-grade pulmonary myxoid liposarcoma. Interact Cardiovasc Thorac Surg 2005;4:402-3.

6. Okby NT, Travis WD. Liposarcoma of the pleural cavity: Clinical and pathologic features of 4 cases with a review of the literature. Arch Pathol Lab Med 2000;124:699-703.

7. Achir A, Ouadnouni Y, Smahi M, Bouchikh M, Msougar Y, Benosman A. Primary pulmonary liposarcoma - A case report. Thorac Cardiovase Surg 2009;57:119-20.

8. Triantopoulou C, Kolliakou E, Karoumpalis I, Yarmenitis S, Dervenis C. Metastatic disease to the pancreas: An imaging challenge. Insights Imaging 2012;3:165-72.

9. Wong WW, Pluth JR, Grado GL, Schild SE, Sanderson DR. Liposarcoma of the pleura. Mayo Clin Proc 1994;69:882-5.

10. Patel SR, Burgess MA, Plager C, Papadopoulos NE, Linke KA, Benjamin RS. Myxoid liposarcoma. Experience with chemotherapy. Cancer 1994;74:1265-9. 